\title{
Quantum entropy for the fuzzy sphere and its monopoles
}

\author{
Nirmalendu Acharyya, ${ }^{a}$ Nitin Chandra $^{b}$ and Sachindeo Vaidya ${ }^{a}$ \\ ${ }^{a}$ Centre for High Energy Physics, Indian Institute of Science, \\ C.V. Raman Avenue, Bangalore-560012, India \\ ${ }^{b}$ The Institute of Mathematical Sciences, \\ C.I.T. Campus, Taramani, Chennai-600113, India \\ E-mail: nirmalendu@cts.iisc.ernet.in, nitinc@imsc.res.in, \\ vaidya@cts.iisc.ernet.in
}

ABSTRACT: Using generalized bosons, we construct the fuzzy sphere $S_{F}^{2}$ and monopoles on $S_{F}^{2}$ in a reducible representation of $\mathrm{SU}(2)$. The corresponding quantum states are naturally obtained using the GNS-construction. We show that there is an emergent nonabelian unitary gauge symmetry which is in the commutant of the algebra of observables. The quantum states are necessarily mixed and have non-vanishing von Neumann entropy, which increases monotonically under a bistochastic Markov map. The maximum value of the entropy has a simple relation to the degeneracy of the irreps that constitute the reducible representation that underlies the fuzzy sphere.

KEYwords: Solitons Monopoles and Instantons, Non-Commutative Geometry

ARXIV EPRINT: 1405.6471 


\section{Contents}

1 Introduction 1

2 A brief review - fuzzy sphere and associated line bundle 3

3 Generalized bosonic oscillators 4

3.1 Schwinger construction with generalized Bose operators 5

4 Fuzzy line bundle with GBO $\quad 8$

$\begin{array}{llr}5 & \text { Mixed states } & \mathbf{1 0}\end{array}$

$\begin{array}{lll}5.1 & \text { Monopole states are mixed } & 13\end{array}$

$\begin{array}{lr}\text { A Basis vectors for } \mathcal{H}_{n l} & 14\end{array}$

\section{Introduction}

Fuzzy spaces are some of the simplest noncommutative geometries. Usually these spaces are described by the algebra of the linear operators on the representation spaces of a Lie group. If the the Lie group is compact, the representations of the group are finite dimensional. In such cases the fuzzy spaces are finite dimensional matrix algebra on which the group acts in a simple way.

For example, the fuzzy 2 -sphere $S_{F}^{2}$ is described by the matrix algebra on the spin- $\mathcal{J}$ representation of the $\mathrm{SU}(2)[1,2]$. The elements of this algebra are the identity $\mathbb{I}_{(2 \mathcal{J}+1)}$, the generators of $\mathrm{SU}(2) \hat{x}_{i}(i=1,2,3)$ and products of these generators. More precisely, the matrix algebra

$$
\left[\hat{x}_{i}, \hat{x}_{j}\right]=i \epsilon_{i j k} \hat{x}_{k}, \quad \hat{x}_{i}^{\dagger}=\hat{x}_{i}, \quad \hat{x}_{i} \hat{x}_{i}=\mathcal{J}(\mathcal{J}+1) \mathbb{I}_{(2 \mathcal{J}+1)}, \quad i, j, k=1,2,3 .
$$

is sufficient describe the fuzzy sphere $S_{F}^{2}$.

Field theories on such a compact fuzzy space are finite dimensional and can naturally incorporate topological objects like instantons and axial anomalies. Hence the nontrivial field configurations on such spaces, especially the classical topological objects like solitons, instantons and monopoles have been the subject of paricular interest to many (for example see $[3-10])$.

These spaces emerge naturally in the matrix models describing branes (see for instance [11-15] and references therein). An particularly interesting model of this type was first discussed in [16]. Roughly speaking, it describes a three-matrix model coupled to a background Ramond-Ramond 4-form field and is described by the action

$$
S=T_{0} \operatorname{Tr}\left[\frac{1}{2} \dot{\phi}_{i}^{2}+\frac{1}{4}\left[\phi_{i}, \phi_{j}\right]^{2}-\frac{i}{3} \kappa \epsilon_{i j k} \phi_{i}\left[\phi_{j}, \phi_{k}\right]\right] \text {. }
$$


$\kappa$ is a (Chern-Simons) coupling constant and $\phi_{i}$ 's $(i=1,2,3)$ are $N \times N$ matrices. The importance of classical nonpertubative solutions like flux tubes, instantons etc in these matrix models are also emphasized in [17].

In absence of the Chern-Simons term (i.e. when $\kappa=0$ ), the potential is extremized by $\left[\phi_{i}, \phi_{j}\right]=0$. This extremum represents $N D 0$-branes. But in presence of the ChernSimons term, there are other extrema of lower energy. These extrema are given by a set of noncommuting matrices

$$
\phi_{i}=\kappa \hat{x}_{i}, \quad\left[\hat{x}_{i}, \hat{x}_{j}\right]=i \epsilon_{i j k} \hat{x}_{k} \quad \text { for } i, j, k=1,2,3 .
$$

Here, $\hat{x}_{i}$ 's are $N \times N$ matrices satisfying $\hat{x}_{i} \hat{x}_{i}=$ fixed and thus describes the algebra of $S_{F}^{2}$ on a spin- $\mathcal{J}\left(\equiv \frac{N-1}{2}\right)$ representation of $\mathrm{SU}(2)$. For the model (1.2), this fuzzy sphere solution can either be an irreducible or reducible representation. If the representation is reducible (i.e. $N \times N$ matrices are block diagonal with the blocks of smaller size), the classical energy $E_{n c}^{r}$ is higher than that of the irreducible one $E_{n c}^{i r}$.

A careful analysis of stability done in [18] shows that all these extremas (both the reducible and the irreducible) are stable. This is a puzzling circumstance as the fuzzy spheres in the reducible representation have higher energy. In $[18,19]$, the puzzle is resolved by taking non-spherical marginal deformations around the fuzzy spheres in reducible representation.

In this article, we investigate this problem from a completely different perspective. When the irreps in the reducible representation are identical, all fuzzy spheres in various irreps have the same "radius" (see table 1 in [18]). Thus it is possible to identify the reducible algebra with a single fuzzy sphere in a reducible representation. We construct these fuzzy spheres by Schwinger construction using Brandt-Greenberg [20] or generalized Bose oscillators. In [21], it was shown that these play a vital role in the construction of classical topological solutions in noncommutative spaces. Here we show that these oscillators give the monopoles on the background of the fuzzy sphere with reducible representation. Further we construct the quantum states corresponding to these fuzzy spheres in reducible representations using GNS construction. We show that there is a gauge symmetry which is related to the number of copies of identical irreps in the reducible representation. A remarkable consequence is that these states are not pure, and hence carry intrinsic quantum entropy $[22,23]$. Such entropy persists at zero temperature, and we show that the entropy can be macroscopically large.

The article is organized as follows. In section 2, we briefly review the description of fuzzy sphere algebra using the standard Schwinger construction and the technique developed in [3] to describe the sections of the complex line bundle on the fuzzy sphere. In section 3 we review the Brandt-Greenberg oscillators in detail and in 3.1 the Schwinger construction with these oscillators. We show that the reducible representation thus obtained describe a fuzzy 2 -sphere. In section 4 we construct the associated line bundles on the $S_{F}^{2}$ corresponding to these unstable vacua using the prescription in [3]. In section 5, we construct the quantum states of the fuzzy sphere in the reducible representation. We show that these have a $U(r)$ gauge symmetry $(r$ is the number of identical irreps in the reducible rep- 
resentation) and are impure. Similarly, the states of the monopole have a $U\left(r^{2}\right)$ gauge symmetry and are impure as well. We compute the entropy associated with these impure states.

\section{A brief review - fuzzy sphere and associated line bundle}

There is a natural chain of descent $\mathbb{C}_{F}^{2} \rightarrow S_{F}^{3} \rightarrow S_{F}^{2}$. The algebra of $\mathbb{C}_{F}^{2}$ is described by the algebra of a pair of independent harmonic oscillators (see [24])

$$
\left[\hat{a}_{\alpha}, \hat{a}_{\beta}^{\dagger}\right]=\delta_{\alpha \beta}, \quad \alpha, \beta=1,2 .
$$

These oscillators acts on the Fock space $\mathcal{F}=\operatorname{span}\left\{\left|n_{1}, n_{2}\right\rangle\right\}$. These are eigenstates of $\hat{N}=\sum_{\alpha} \hat{a}_{\alpha}^{\dagger} \hat{a}_{\alpha}$.

The operator $\hat{\chi}_{\alpha}=\hat{a}_{\alpha} \frac{1}{\sqrt{\hat{N}}}$ is well defined on $\mathcal{F}$ except for the state $|0,0\rangle$. As $\hat{\chi}_{\alpha}^{\dagger} \hat{\chi}_{\alpha}=1$, the algebra generated by $\hat{\chi}_{\alpha}$ describes the fuzzy 3 -sphere $S_{F}^{3}$.

The Schwinger construction

$$
\hat{x}_{i}=\frac{1}{2} \hat{a}_{\alpha}^{\dagger}\left(\sigma_{i}\right)_{\alpha \beta} \hat{a}_{\beta}, \quad\left[\hat{x}_{i}, \hat{x}_{j}\right]=i \epsilon_{i j k} \hat{x}_{k}, \quad \hat{x}_{i} \hat{x}_{i}=\frac{\hat{N}}{2}\left(\frac{\hat{N}}{2}+1\right) .
$$

is the noncommutative version of Hopf map [25].

In the subspace $\mathcal{F}_{n}=\operatorname{span}\left\{\left|n_{1}, n_{2}\right\rangle: n_{1}+n_{2}=n\right\} \subset \mathcal{F}$, the operator $\hat{x}_{i} \hat{x}_{i}=$ $\frac{n}{2}\left(\frac{n}{2}+1\right)=$ fixed. Thus this subspace is the carrier space of $(n+1)$ dimensional UIR of $\mathrm{SU}(2)$.

The operators

$$
\hat{q}_{i}=\frac{1}{\hat{N}} \hat{x}_{i}
$$

generate the algebra of the fuzzy sphere $S_{F}^{2}$. The map

$$
\hat{q}_{i}=\frac{1}{2} \hat{\chi}_{\alpha}^{\dagger}\left(\sigma_{i}\right)_{\alpha \beta} \hat{\chi}_{\beta}
$$

from $S_{F}^{3} \rightarrow S_{F}^{2}$ is the noncommutative analogue of the Hopf fibration.

The associated complex line bundles are given by complex scalar fields $\Phi$ with a topological charge. These scalar fields map $\mathcal{F}_{n} \rightarrow \mathcal{F}_{l}$, where the topological charge is $\kappa=$ $\frac{1}{2}(l-n)$. We can find a basis for these $\Phi$ exploiting the group theoretic properties of $S_{F}^{2}[3]$.

In general, $\Phi$ 's are $(l+1) \times(n+1)$ rectangular matrices and are element of a noncommutative bi-module $\mathcal{H}_{n l}$ - it is a left $\mathcal{A}_{l}$-module and a right $\mathcal{A}_{n}$-module. On $\mathcal{H}_{n l}$, the adjoint of $\hat{x}_{i}$ acts as

$$
A d\left(\hat{x}_{i}\right) \Phi=\left[\hat{x}_{i}^{(l)} \Phi-\Phi \hat{x}_{i}^{(n)}\right], \quad \Phi \in \mathcal{H}_{n l},
$$

and generates rotation

$$
\left[A d\left(\hat{x}_{i}\right), A d\left(\hat{x}_{j}\right)\right]=i \epsilon_{i j k} A d\left(\hat{x}_{k}\right) .
$$

This action of the $\mathrm{SU}(2)$ gives the direct product $\frac{l}{2} \otimes \frac{n}{2}$ of the two UIRs $\frac{l}{2}$ and $\frac{n}{2}$. The elements of $\mathcal{H}_{n l}$ can therefore be expanded in terms of the eigenfunctions $\Psi_{J, \kappa, m}^{j}$ of $A d\left(\hat{x}_{3}\right)$ and $\left[A d\left(\hat{x}_{i}\right) A d\left(\hat{x}_{i}\right)\right]$ belonging to the irreducible representations in the decomposition of $\frac{l}{2} \otimes \frac{n}{2}$ :

$$
\frac{l}{2} \otimes \frac{n}{2}=\kappa \oplus(\kappa+1) \oplus \ldots \oplus J, \quad \text { where } J \equiv \frac{l+n}{2} .
$$


An arbitrary element $\Phi$ of $\mathcal{H}_{n l}$ can be expressed as

$$
\Phi=\sum_{j=\kappa}^{J} \sum_{m=-j}^{j} c_{J, \kappa, m}^{j} \Psi_{J, \kappa, m}^{j}, \quad c_{J, \kappa, m}^{j} \in \mathbb{C} .
$$

The topological charge operator $\hat{K}_{0}$ is

$$
\hat{K}_{0} \equiv \frac{1}{2}[\hat{N},]
$$

and it satisfies

$$
\left[A d\left(\hat{x}_{3}\right), \hat{K}_{0}\right]=0=\left[A d\left(\hat{x}_{i}\right) A d\left(\hat{x}_{i}\right), \hat{K}_{0}\right]
$$

Any element $\Phi$ of $\mathcal{H}_{n l}$ is also an eigenfunction of $\hat{K}_{0}$ :

$$
\hat{K}_{0} \Phi=\frac{\kappa}{2} \Phi
$$

$\Phi$ is thus the noncommutative analogue of a section of the complex line bundle with topological charge $\kappa$.

\section{$3 \quad$ Generalized bosonic oscillators}

The generalized bosonic oscillators [20] change the number of quanta by a positive integer $K$. We briefly recall their construction.

Consider a standard representation of bosonic oscillator $(\hat{a}, \mathbb{H})$ :

$$
\left[\hat{a}, \hat{a}^{\dagger}\right]=1 .
$$

The Hilbert space $\mathbb{H}$ is spanned by the basis of the eigenvectors of the number operator $\hat{N} \equiv \hat{a}^{\dagger} \hat{a}$ :

$$
\mathbb{H}=\left\{c_{n}|n\rangle, n=0,1, \cdots, \infty\right\}, \quad \hat{N}|n\rangle=n|n\rangle .
$$

$\mathbb{H}$ can be split into two disjoint subspaces $\mathbb{H}_{e}=\left\{\sum c_{2 n}|2 n\rangle \in \mathbb{H}\right\}$ and $\mathbb{H}_{o}=$ $\left\{\sum c_{2 n+1}|2 n+1\rangle \in \mathbb{H}\right\}$. (The labels $e$ and $o$ stand for even and odd respectively.) The (projection) operators

$$
\Lambda^{e}=\sum_{n=0}^{\infty}|2 n\rangle\left\langle 2 n\left|, \quad \Lambda^{o}=\sum_{n=0}^{\infty}\right| 2 n+1\right\rangle\langle 2 n+1|
$$

project onto the subspaces $\mathbb{H}_{e}$ and $\mathbb{H}_{o}$ respectively. On $\mathbb{H}_{e}$ and $\mathbb{H}^{o}$, one can define operators $\hat{b}^{e}$ and $\hat{b}^{o}$ as

$$
\begin{aligned}
& \hat{b}^{e}|2 n\rangle=n^{\frac{1}{2}}|2 n-2\rangle, \quad \hat{b}^{e \dagger}|2 n\rangle=(n+1)^{\frac{1}{2}}|2 n+2\rangle, \quad \hat{b}^{e}|0\rangle=0 . \\
& \hat{b}^{o}|2 n+1\rangle=n^{\frac{1}{2}}|2 n-1\rangle, \quad \hat{b}^{o \dagger}|2 n+1\rangle=(n+1)^{\frac{1}{2}}|2 n+3\rangle, \quad \hat{b}^{o}|1\rangle=0 .
\end{aligned}
$$


These operators satisfy $\left[\hat{b}^{e}, \hat{b}^{e \dagger}\right]=1$ and $\left[\hat{b}^{o}, \hat{b}^{o \dagger}\right]=1$. Thus as representations $\left(\hat{b}^{o}, \mathbb{H}_{o}\right)$, $\left(\hat{b}^{e}, \mathrm{H}_{e}\right)$ and $(\hat{a}, \mathbb{H})$ are isomorphic to each other. ${ }^{1}$ In other words, there exist unitary operators $U_{e}$ and $U_{o}$ such that $U_{e}^{\dagger} \hat{b}^{e} U_{e}=\hat{a}$ and $U_{o}^{\dagger} \hat{b}^{o} U_{o}=\hat{a}$.

Using $\Lambda^{e}$ and $\Lambda^{o}$ of (3.2), one can define an operator $\hat{b}$

$$
\hat{b}=\hat{b}^{e} \Lambda^{e}+\hat{b}^{o} \Lambda^{o}
$$

annihilates $\left(\hat{b}^{\dagger}\right.$ creates) two quanta on $\mathbb{H}$. (Notice that both $|0\rangle$ and $|1\rangle$ are annihilated by $\hat{b}$.) $\hat{b}$ satisfies $\left[\hat{b}, \hat{b}^{\dagger}\right]=1$ and thus $(\hat{b}, \mathbb{H})$ forms a reducible representation of the oscillator algebra, with (3.5) as its decomposition into irreducibles.

The above can be generalized to construct an operator $\hat{b}^{(K)}$ which lowers a state $|n\rangle$ by $K$ steps. We start by defining projection operators $\Lambda^{i}$ by

$$
\Lambda^{i}=\sum_{n=0}^{\infty}|K n+i\rangle\langle K n+i|, \quad i=0,1, \cdots K-1 .
$$

that project onto subspaces $\mathbb{H}_{i}=\left\{\sum_{n} c_{K n+i}|K n+i\rangle \in \mathbb{H}\right\}$. In each $\mathbb{H}_{i}$, we define operators $\hat{b}^{i}$ and $\hat{b}^{i \dagger}$ that satisfy $\left[\hat{b}^{i}, \hat{b}^{i \dagger}\right]=1$ and hence correspond to the UIR of the oscillator algebra. A reducible representation is given by

$$
\hat{b}^{(K)}=\sum_{i=0}^{K-1} \hat{b}^{i} \Lambda^{i}, \quad \hat{b}^{i}|K n+i\rangle=\sqrt{n}|K n+i-K\rangle, \quad \mathbb{H}=\oplus_{i=0}^{K-1} \mathbb{H}_{i}
$$

with $\left[\hat{b}^{(K)}, \hat{b}^{(K) \dagger}\right]=1$. Again, $\left(\hat{b}^{i}, \mathbb{H}_{i}\right)$ is isomorphic to $(\hat{a}, \mathbb{H})$ and $\left(\hat{b}^{(K)}, \mathbb{H}\right)$ forms a reducible representation of the oscillator algebra.

The equations (3.5) represent the case $K=2$, the simplest non-trivial example of this construction. Henceforth we will use $\hat{b}$ for $\hat{b}^{(2)}$. An explicit expression for $\hat{b}$ is [26]

$$
\hat{b}=\frac{1}{\sqrt{2}}\left(\hat{a} \frac{1}{\sqrt{\hat{N}}} \hat{a} \Lambda^{e}+\hat{a} \frac{1}{\sqrt{\hat{N}+1}} \hat{a} \Lambda^{o}\right) .
$$

\subsection{Schwinger construction with generalized Bose operators}

By using the $\hat{b}^{K}$ 's of the previous section in the Schwinger construction, we get reducible representations of $\mathrm{SU}(2)$. This will have non-trivial implications in the construction of the line bundles on the fuzzy sphere. Let us briefly see this.

The fuzzy space $\mathbb{C}_{F}^{2}$ is described by two independent oscillators which acts on the space $\mathcal{F}$

$$
\mathcal{F}=\mathrm{H} \otimes \mathbb{H}
$$

This space can be spanned by the eigenstates of the number operator $\hat{N}=\sum_{\alpha} \hat{a}_{\alpha}^{\dagger} \hat{a}_{\alpha}$ :

$$
\mathcal{F}=\operatorname{span}\left\{\left|n_{1}, n_{2}\right\rangle: \hat{N}_{\alpha}\left|n_{1}, n_{2}\right\rangle=n_{\alpha}\left|n_{1}, n_{2}\right\rangle, \alpha=1,2\right\}
$$

where $\left|n_{1}, n_{2}\right\rangle \equiv\left|n_{1}\right\rangle \otimes\left|n_{2}\right\rangle$.

\footnotetext{
${ }^{1}$ Note that all these operators are unbounded and are hence defined on a dense domains in $\mathbb{H}$. A detailed discussion about these domains can be found in [20].
} 
$\mathcal{F}$ can now be split into four subspaces

$$
\left.\begin{array}{l}
\mathcal{F}^{e e}=\mathbb{H}_{e} \otimes \mathbb{H}_{e}=\operatorname{span}\left\{\left|2 n_{1}, 2 n_{2}\right\rangle\right\} \\
\mathcal{F}^{e o}=\mathbb{H}_{e} \otimes \mathbb{H}_{o}=\operatorname{span}\left\{\left|2 n_{1}, 2 n_{2}+1\right\rangle\right\} \\
\mathcal{F}^{o e}=\mathbb{H}_{o} \otimes \mathbb{H}_{e}=\operatorname{span}\left\{\left|2 n_{1}+1,2 n_{2}\right\rangle\right\} \\
\mathcal{F}^{o o}=\mathbb{H}_{o} \otimes \mathbb{H}_{o}=\operatorname{span}\left\{\left|2 n_{1}+1,2 n_{2}+1\right\rangle\right\}
\end{array}\right\} \quad \text { i.e. } \mathcal{F}=\oplus_{\lambda} \mathcal{F}^{\lambda}
$$

where $\lambda=e e, e o, o e, o o$. The projectors

$$
\begin{aligned}
& \Lambda_{e e}=\Lambda_{1}^{e} \Lambda_{2}^{e}, \quad \Lambda_{e o}=\Lambda_{1}^{e} \Lambda_{2}^{o}, \quad \Lambda_{o e}=\Lambda_{1}^{o} \Lambda_{2}^{e}, \quad \Lambda_{o o}=\Lambda_{1}^{o} \Lambda_{2}^{o}, \\
& \sum_{\lambda} \Lambda_{\lambda}=1, \quad \Lambda_{\lambda} \Lambda_{\lambda^{\prime}}=\Lambda_{\lambda} \delta_{\lambda \lambda^{\prime}}
\end{aligned}
$$

project to subspaces of the two oscillator Hilbert space: $\Lambda_{\lambda}$ projects to subspace $\mathcal{F}^{\lambda}$. $\left(\Lambda_{\alpha}^{e}\right.$ and $\Lambda_{\alpha}^{o}$ are the projectors defined in (3.2).) Explicitly

$$
\Lambda_{e e}=\cos ^{2} \frac{\hat{N}_{1} \pi}{2} \cos ^{2} \frac{\hat{N}_{2} \pi}{2}, \quad \Lambda_{e o}=\cos ^{2} \frac{\hat{N}_{1} \pi}{2} \sin ^{2} \frac{\hat{N}_{2} \pi}{2} \quad \text { etc. }
$$

Consider two independent generalized bosonic oscillator $\hat{b}_{1}$ and $\hat{b}_{2}$ :

$$
\hat{b}_{\alpha}=\hat{b}_{\alpha}^{e} \Lambda_{\alpha}^{e}+\hat{b}_{\alpha}^{o} \Lambda_{\alpha}^{o}, \quad \alpha=1,2
$$

where

$$
\hat{b}_{\alpha}^{e}=\frac{1}{\sqrt{2}} \hat{a}_{\alpha} \frac{1}{\sqrt{\hat{N}_{\alpha}}} \hat{a}_{\alpha}, \quad \hat{b}_{\alpha}^{o}=\frac{1}{\sqrt{2}} \hat{a}_{\alpha} \frac{1}{\sqrt{\hat{N}_{\alpha}+1}} \hat{a}_{\alpha}, \quad \hat{N}_{\alpha}=\hat{a}_{\alpha}^{\dagger} \hat{a}_{\alpha} .
$$

$\hat{b}_{1}^{e}$ only acts on the states in $\mathcal{F}^{e e} \oplus \mathcal{F}^{e o}$ and so on. Let us define a set of operators

$$
\hat{\xi}_{\alpha}=\hat{b}_{\alpha} \frac{1}{\sqrt{\hat{M}}}, \quad \alpha=1,2, \quad \hat{M}=\hat{b}_{1}^{\dagger} \hat{b}_{1}+\hat{b}_{2}^{\dagger} \hat{b}_{2} .
$$

The operator $\hat{M}$

$$
\hat{M}=\frac{1}{2}\left(\hat{N}-\Lambda_{1}^{o}-\Lambda_{2}^{o}\right)
$$

vanishes when acted on the states $|0,0\rangle,|0,1\rangle,|1,0\rangle$ and $|1,1\rangle$. We exclude these states from the domain of $\hat{\xi}_{\alpha}$ so that $\hat{\xi}_{\alpha}$ are well-defined. These operators satisfy

$$
\hat{\xi}_{\alpha}^{\dagger} \hat{\xi}_{\alpha}=1
$$

and hence defines the fuzzy 3 -sphere $S_{F}^{3}$.

We define the operator map

$$
\begin{aligned}
\hat{t}_{i} & =\frac{1}{2} \hat{\xi}_{\alpha}^{\dagger}\left(\sigma_{i}\right)_{\alpha \beta} \hat{\xi}_{\beta}=\frac{1}{\hat{M}} \hat{s}_{i}, \quad \hat{s}_{i}=\frac{1}{2} \hat{b}^{\dagger} \sigma_{i} \hat{b}, \quad i=1,2,3 \\
\text { where } \quad \hat{b} & =\left(\begin{array}{c}
\hat{b}_{1} \\
\hat{b}_{2}
\end{array}\right) \quad \text { and } \sigma_{i}=\text { Pauli matrices. }
\end{aligned}
$$


They satisfy

$$
\left[\hat{s}_{i}, \hat{s}_{j}\right]=i \epsilon_{i j k} \hat{s}_{k}, \quad\left[\hat{s}_{k}, \hat{N}\right]=0=\left[\hat{s}_{k}, \hat{M}\right] .
$$

The "Casimir" can be expressed as

$$
\hat{s}_{i} \hat{s}_{i}=\frac{\hat{M}}{2}\left(\frac{\hat{M}}{2}+1\right), \quad \hat{t}_{i} \hat{t}_{i}=\frac{1}{2}\left(\frac{1}{2}+\frac{1}{\hat{M}}\right) .
$$

Let us define the space $\mathcal{G}_{n}$

$$
\mathcal{G}_{n}=\mathcal{F}_{2 n}^{e e} \oplus \mathcal{F}_{2 n+1}^{e o} \oplus \mathcal{F}_{2 n+1}^{o e} \oplus \mathcal{F}_{2 n+2}^{o o}, \quad \mathcal{G}_{n} \subset \mathcal{F}
$$

In this subspace, $\hat{M}$ takes the value $n$ and hence in $\mathcal{G}_{n}, \hat{t}_{i} \hat{t}_{i}$ is fixed:

$$
\hat{t}_{i} \hat{t}_{i}=\frac{1}{2}\left(\frac{1}{2}+\frac{1}{n}\right)
$$

The algebra generated by $\hat{t}_{i}$ restricted to $\mathcal{G}_{n}$ is the fuzzy 2 -sphere $S_{F}^{2}$ and the JordanSchwinger construction (3.20) is the fuzzy analogue of the the Hopf map: $S_{F}^{3} \rightarrow S_{F}^{2}$.

In the commutative limit $n \rightarrow \infty$,

$$
\lim _{n \rightarrow \infty} \hat{t}_{i} \hat{t}_{i}=\lim _{n \rightarrow \infty} \frac{1}{2}\left(\frac{1}{2}+\frac{1}{n}\right)=\frac{1}{4}
$$

Here the "radius" does not depend on $n$ and we recover the standard $S^{2}$.

In $\mathcal{G}_{n}$, the operators $\hat{s}_{i}$ create and destroy same number of quanta and hence $\hat{s}_{i}: \mathcal{G}_{n} \rightarrow$ $\mathcal{G}_{n}$. These are the generators of rotations in $\mathcal{G}_{n}$. Using (3.5), we can decompose $\hat{s}_{i}$ as follows:

$$
\hat{s}_{i}=\hat{s}_{i}^{e e} \Lambda_{e e}+\hat{s}_{i}^{e o} \Lambda_{e o}+\hat{s}_{i}^{o e} \Lambda_{o e}+\hat{s}_{i}^{o o} \Lambda_{o o}
$$

Each $\hat{s}_{i}^{\lambda}$ satisfy

$$
\left[\hat{s}_{i}^{\lambda}, \hat{s}_{j}^{\lambda}\right]=i \epsilon_{i j k} \hat{s}_{k}^{\lambda}
$$

and we obtain the following table:

$$
\begin{array}{|c|l|}
\hline \mathcal{F}_{2 n}^{e e} & \hat{s}_{i}^{e e} \hat{s}_{i}^{e e}=\frac{n}{2}\left(\frac{n}{2}+1\right) \\
\hline \mathcal{F}_{2 n+1}^{e O} & \hat{s}_{i}^{e o} \hat{s}_{i}^{e O}=\frac{n}{2}\left(\frac{n}{2}+1\right) \\
\hline \mathcal{F}_{2 n+1}^{o e} & \hat{s}_{i}^{o e} \hat{s}_{i}^{o e}=\frac{n}{2}\left(\frac{n}{2}+1\right) \\
\hline \mathcal{F}_{2 n+2}^{o O} & \hat{s}_{i}^{O O} \hat{s}_{i}^{o O}=\frac{n}{2}\left(\frac{n}{2}+1\right) . \\
\hline
\end{array}
$$

The subspaces $\mathcal{F}_{2 n}^{e e}, \mathcal{F}_{2 n+1}^{e o}, \mathcal{F}_{2 n+1}^{o e}$ and $\mathcal{F}_{2 n+2}^{o o}$ are all $(n+1)$-dimensional, and each of them is the carrier space of $(n+1)$-dimensional UIR of $\mathrm{SU}(2)$. 
On the other hand $\mathcal{G}_{n}$ is a $(4 n+4)$-dimensional space and is the carrier space of the reducible representation of $\mathrm{SU}(2)$ generated by $\hat{s}_{i}$ with the irreducible decomposition (3.27). In this space, $\hat{s}_{i}$ can be represented by $(4 n+4) \times(4 n+4)$ block diagonal matrices:

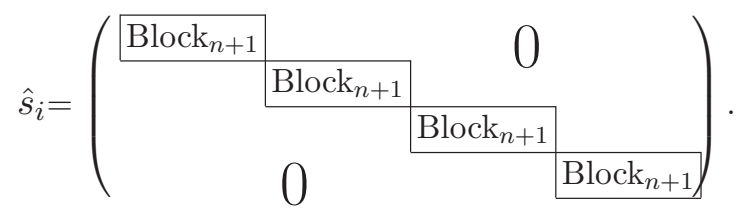

\section{Fuzzy line bundle with GBO}

$\mathcal{H}_{n l}$ is the space of linear maps $\Phi: \mathcal{G}_{n} \rightarrow \mathcal{G}_{l}$, representated by $(4 l+4) \times(4 n+4)$ matrices. It is a left $\mathcal{A}_{l}$-module and a right $\mathcal{A}_{n}$-module $\left(\mathcal{A}_{l}\right.$ and $\mathcal{A}_{n}$ were defined in section 2$)$.

On $\mathcal{H}_{n n}$, the adjoint action of $s_{i}^{(n)}\left(\hat{s}_{i}^{(n)}\right.$ are the restriction of the operators $\hat{s}_{i}$ to $\left.\mathcal{G}_{n}\right)$ generates rotations:

$$
A d\left(\hat{s}_{i}\right) \Phi \equiv \hat{S}_{i} \Phi=\left[\hat{s}_{i}^{(n)}, \Phi\right], \quad \Phi \in \mathcal{H}_{n n} .
$$

In $\mathcal{H}_{n n}, \hat{S}_{i}$ defined above satisfies

$$
\left[\hat{S}_{i}, \hat{S}_{j}\right]=i \epsilon_{i j k} \hat{S}_{k}
$$

In $\mathcal{H}_{n l}$, the generators of the $\mathrm{SU}(2)$ act as

$$
\hat{S}_{i} \Phi \equiv \hat{s}_{i}^{(l)} \Phi-\Phi \hat{s}_{i}^{(n)}, \quad \Phi \in \mathcal{H}_{n l}
$$

and satisfy (4.2). The $\hat{S}_{i}$ acting on $\mathcal{H}_{n l}$ correspond to the following reducible representation of $\mathrm{SU}(2)$ :

$$
\left(\frac{l}{2} \oplus \frac{l}{2} \oplus \frac{l}{2} \oplus \frac{l}{2}\right) \otimes\left(\frac{n}{2} \oplus \frac{n}{2} \oplus \frac{n}{2} \oplus \frac{n}{2}\right),
$$

which in turn can be decomposed into UIRs as

$$
[k \oplus(k+1) \ldots \oplus J] \oplus[k \oplus(k+1) \ldots \oplus J] \ldots 16 \text { copies },
$$

\begin{tabular}{|c|c|}
\hline $\begin{array}{cc}\mathcal{F}_{2 n} & \mathcal{F}_{2 l} \\
\mathcal{F}_{2 n+1} & \mathcal{F}_{2 l+1} \\
\mathcal{F}_{2 n+1} & \mathcal{F}_{2 l+1} \\
\mathcal{F}_{2 n+2} & \mathcal{F}_{2 l+2}\end{array}$ & 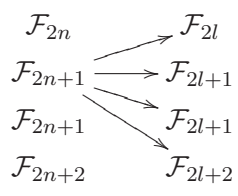 \\
\hline $\begin{array}{l}\mathcal{F}_{2 n} \\
\mathcal{F}_{2 n+1} \longrightarrow \mathcal{F}_{2 l+1} \\
\mathcal{F}_{2 n+1} \longrightarrow \mathcal{F}_{2 l+1} \\
\mathcal{F}_{2 n+2} \longrightarrow \mathcal{F}_{2 l+2}\end{array}$ & $\begin{array}{c}\mathcal{F}_{2 n} \\
\mathcal{F}_{2 n+1} \\
\mathcal{F}_{2 n+1} \\
\mathcal{F}_{2 n+2} \longrightarrow \mathcal{F}_{2 l}\end{array}$ \\
\hline
\end{tabular}

with $k=\frac{|l-n|}{2}$ and $J=\frac{l+n}{2}$.

These sixteen identical copies of $\frac{l}{2} \otimes \frac{n}{2}$ essentially correspond to the following sixteen types of maps $\Phi: \mathcal{G}_{n} \longrightarrow \mathcal{G}_{l}$ : 
The maps $\Phi \in \mathcal{H}_{n l}$ can be expanded in the basis of the eigenfunctions of $\hat{S}_{i} \hat{S}_{i}$ and $\hat{S}_{3}$ belonging to the irreducible representations in decomposition of (4.5). We can explicitly find the basis vectors by constructing the highest weight vectors, and by repeated action of $\hat{S}_{-}\left(\equiv \hat{S}_{1}-i \hat{S}_{2}\right)$. Below we list all the highest weight vector (details for a specific example are in the appendix A):

\begin{tabular}{|c|c|}
\hline & Highest weight function \\
\hline $\mathcal{F}_{2 n}^{e e} \rightarrow \mathcal{F}_{2 l}^{e e}$ & $\left(\Psi^{e e \rightarrow e e}\right)_{j, j}^{J k}=\left(\hat{b}_{1}^{e \dagger}\right)^{\tilde{l}}\left(\hat{b}_{2}^{e}\right)^{\tilde{n}} \Lambda_{e e}$ \\
\hline $\mathcal{F}_{2 n}^{e e} \rightarrow \mathcal{F}_{2 l+1}^{e o}$ & $\left(\Psi^{e e \rightarrow e o}\right)_{j, j}^{J k}=\hat{a}_{2}^{\dagger} \frac{1}{\sqrt{\hat{N}_{2}+1}}\left(\hat{b}_{1}^{e \dagger}\right)^{\tilde{l}}\left(\hat{b}_{2}^{e}\right)^{\tilde{n}} \Lambda_{e e}$ \\
\hline $\mathcal{F}_{2 n}^{e e} \rightarrow \mathcal{F}_{2 l+1}^{o e}$ & $\left(\Psi^{e e \rightarrow o e}\right)_{j, j}^{J k}=\hat{a}_{1}^{\dagger} \frac{1}{\sqrt{\hat{N}_{1}+1}}\left(\hat{b}_{1}^{e \dagger}\right)^{\tilde{l}}\left(\hat{b}_{2}^{e}\right)^{\tilde{n}} \Lambda_{e e}$ \\
\hline $\mathcal{F}_{2 n}^{e e} \rightarrow \mathcal{F}_{2 l+2}^{o o}$ & $\left(\Psi^{e e \rightarrow o o}\right)_{j, j}^{J k}=\hat{a}_{1}^{\dagger} \frac{1}{\sqrt{\hat{N}_{1}+1}} \hat{a}_{2}^{\dagger} \frac{1}{\sqrt{\hat{N}_{2}+1}}\left(\hat{b}_{1}^{e \dagger}\right)^{\tilde{l}}\left(\hat{b}_{2}^{e}\right)^{\tilde{n}} \Lambda_{e e}$ \\
\hline $\mathcal{F}_{2 n+1}^{e o} \rightarrow \mathcal{F}_{2 l}^{e e}$ & $\left(\Psi^{e o \rightarrow e e}\right)_{j, j}^{J k}=\hat{a}_{2} \frac{1}{\sqrt{\hat{N}_{2}}}\left(\hat{b}_{1}^{e \dagger}\right)^{\tilde{l}}\left(\hat{b}_{2}^{o}\right)^{\tilde{n}} \Lambda_{e o}$ \\
\hline $\mathcal{F}_{2 n+1}^{e o} \rightarrow \mathcal{F}_{2 l+1}^{e o}$ & $\left(\Psi^{e o \rightarrow e o}\right)_{j, j}^{J k}=\left(\hat{b}_{1}^{e \dagger}\right)^{\tilde{l}}\left(\hat{b}_{2}^{o}\right)^{\tilde{n}} \Lambda_{e o}$ \\
\hline $\mathcal{F}_{2 n+1}^{e o} \rightarrow \mathcal{F}_{2 l+1}^{o e}$ & $\left(\Psi^{e o \rightarrow o e}\right)_{j, j}^{J k}=\hat{a}_{1}^{\dagger} \frac{1}{\sqrt{\hat{N}_{1}+1}} \hat{a}_{2} \frac{1}{\sqrt{\hat{N}_{2}}}\left(\hat{b}_{1}^{e \dagger}\right)^{\tilde{l}}\left(\hat{b}_{2}^{o}\right)^{\tilde{n}} \Lambda_{e o}$ \\
\hline $\mathcal{F}_{2 n+1}^{e O} \rightarrow \mathcal{F}_{2 l+2}^{o o}$ & $\left(\Psi^{e o \rightarrow o o}\right)_{j, j}^{J k}=\hat{a}_{1}^{\dagger} \frac{1}{\sqrt{\hat{N}_{1}+1}}\left(\hat{b}_{1}^{e \dagger}\right)^{\tilde{l}}\left(\hat{b}_{2}^{o}\right)^{\tilde{n}} \Lambda_{e o}$ \\
\hline $\mathcal{F}_{2 n+1}^{o e} \rightarrow \mathcal{F}_{2 l}^{e e}$ & $\left(\Psi^{o e \rightarrow e e}\right)_{j, j}^{J k}=\hat{a}_{1} \frac{1}{\sqrt{\hat{N}_{1}}}\left(\hat{b}_{1}^{o \dagger}\right)^{\tilde{l}}\left(\hat{b}_{2}^{e}\right)^{\tilde{n}} \Lambda_{o e}$ \\
\hline $\mathcal{F}_{2 n+1}^{o e} \rightarrow \mathcal{F}_{2 l+1}^{e o}$ & $\left(\Psi^{o e \rightarrow e o}\right)_{j, j}^{J k}=\hat{a}_{1} \frac{1}{\sqrt{\hat{N}_{1}}} \hat{a}_{2}^{\dagger} \frac{1}{\sqrt{\hat{N}_{2}+1}}\left(\hat{b}_{1}^{o \dagger}\right)^{\tilde{l}}\left(\hat{b}_{2}^{e}\right)^{\tilde{n}} \Lambda_{o e}$ \\
\hline $\mathcal{F}_{2 n+1}^{o e} \rightarrow \mathcal{F}_{2 l+1}^{o e}$ & $\left(\Psi^{o e \rightarrow o e}\right)_{j, j}^{J k}=\left(\hat{b}_{1}^{o \dagger}\right)^{\tilde{l}}\left(\hat{b}_{2}^{e}\right)^{\tilde{n}} \Lambda_{o e} \frac{(\tilde{l}+\tilde{n})}{2}$ \\
\hline $\mathcal{F}_{2 n+1}^{o e} \rightarrow \mathcal{F}_{2 l+2}^{o o}$ & $\left(\Psi^{o e \rightarrow o o}\right)_{j, j}^{J k}=\hat{a}_{2}^{\dagger} \frac{1}{\sqrt{\hat{N}_{2}+1}}\left(\hat{b}_{1}^{o \dagger}\right)^{\tilde{l}}\left(\hat{b}_{2}^{e}\right)^{\tilde{n}} \Lambda_{o e}$ \\
\hline $\mathcal{F}_{2 n+2}^{o o} \rightarrow \mathcal{F}_{2 l}^{e e}$ & $\left(\Psi^{o o \rightarrow e e}\right)_{j, j}^{J k}=\hat{a}_{1} \frac{1}{\sqrt{\hat{N}_{1}}} \hat{a}_{2} \frac{1}{\sqrt{N_{2}}}\left(\hat{b}_{1}^{o \dagger}\right)^{\tilde{l}}\left(\hat{b}_{2}^{o}\right)^{\tilde{n}}$ \\
\hline $\mathcal{F}_{2 n+2}^{o O} \rightarrow \mathcal{F}_{2 l+1}^{e O}$ & $\left(\Psi^{o o \rightarrow e o}\right)_{j, j}^{J k}=\hat{a}_{1} \frac{1}{\sqrt{\hat{N}_{1}}}\left(\hat{b}_{1}^{o \dagger}\right)^{\tilde{l}}\left(\hat{b}_{2}^{o}\right)^{\tilde{n}}$ \\
\hline $\mathcal{F}_{2 n+2}^{o O} \rightarrow \mathcal{F}_{2 l+1}^{o e}$ & $\left(\Psi^{o o \rightarrow o e}\right)_{j, j}^{J k}=\hat{a}_{2} \frac{1}{{\sqrt{N_{2}}}}\left(\hat{b}_{1}^{o \dagger}\right)^{\tilde{l}}\left(\hat{b}_{2}^{o}\right)^{\tilde{n}}$ \\
\hline $\mathcal{F}_{2 n+2}^{o O} \rightarrow \mathcal{F}_{2 l+2}^{o O}$ & $\left(\Psi^{o o \rightarrow o o}\right)_{j, j}^{J k}=\left(\hat{b}_{1}^{o \dagger}\right)^{\tilde{l}}\left(\hat{b}_{2}^{o}\right)^{\tilde{n}}$ \\
\hline
\end{tabular}

where $0 \leq \tilde{n} \leq n$ and $\tilde{l}-\tilde{n}=l-n=2 k$. 
Each of these highest weight vectors belong to the representation with

$$
j=\frac{1}{2}(\tilde{l}+\tilde{n}) .
$$

The range of $j$ in all the cases is

$$
j=k, k+1, k+2 \ldots J
$$

These are the irreducible representations in (4.5). The other basis vectors can be found by operating the lowering operator suitably, as in (A.9), on these highest weight vectors.

Therefore any arbitrary element of $\mathcal{H}_{n l}$ can be expanded in the basis of these operators as

$$
\Phi=\sum_{\alpha} \sum_{j=k}^{J} \sum_{m=-j}^{j} C_{\alpha}^{j, m}\left(\Psi^{\alpha}\right)_{j, m}^{J k}
$$

where $\alpha=e e \rightarrow e e, e e \rightarrow o o \cdots$.

The topological charge operator $\hat{K}_{0}$ is

$$
\hat{K}_{0} \equiv=\frac{1}{2}[\hat{M}, \quad], \quad \hat{M}=\hat{b}_{1}^{\dagger} \hat{b}_{1}+\hat{b}_{2}^{\dagger} \hat{b}_{2} .
$$

The elements of $\mathcal{H}_{n l}$ satisfy

$$
\hat{K}_{0} \Phi=\frac{k}{2} \Phi, \quad \Phi \in \mathcal{H}_{n l}, \quad k \in \mathbb{Z}^{+} .
$$

Hence $\Phi \in \mathcal{H}_{n l}$ are the noncommutative analogue of the complex line bundles with topological charge $k$.

\section{$5 \quad$ Mixed states}

The algebra $\mathcal{A}$ of the fuzzy sphere is generated by $\left\{\mathbb{I}, \hat{s}_{i}, \hat{s}_{i} \hat{s}_{j}, \cdots\right\}$ where the $\hat{s}_{i}$ were defined in (3.20). This algebra is also a $*$-algebra, with $\hat{s}_{i}^{*}=\hat{s}_{i}$. In general, this algebra is simply the algebra $M_{N+1}(\mathbb{C})$ of $(N+1) \times(N+1)$ matrices. Notice that this includes the description of both the irreducible as well as the reducible fuzzy spheres.

A state on this algebra is a linear map $\omega: \mathcal{A} \rightarrow \mathbb{C}$ satisfying

$$
\omega\left(A^{*} A\right) \geq 0 \quad \forall A \in \mathcal{A}, \quad \omega(\mathbb{I})=1 .
$$

Given a state on a $*$-algebra, we can use the GNS construction to construct the Hilbert space $\mathbb{H}_{G N S}$ to make contact with standard quantum mechanical description. The advantage of this algebraic formalism is that both pure and mixed density matrices may be discussed in the same unified language.

To describe the fuzzy sphere in an algebraic language, we need to impose more conditions on the state $\omega$. To describe the irreducible $S_{F}^{2}$, we require that $\omega\left(\hat{s}_{i} \hat{s}_{i}\right)=$ $\frac{N}{2}\left(\frac{N}{2}+1\right) \omega(\mathbb{I})=\frac{N}{2}\left(\frac{N}{2}+1\right)$. With this condition, the resulting $\mathbb{H}_{G N S}$ is simply the 
unique (upto unitary equivalence) carrier space of the $(N+1)$-dimensional representation of $\mathrm{SU}(2)$.

To describe the reducible representations, consider $n$ projectors $P_{a}^{\left(n_{a}\right)}, a=1, \cdots n$, where $n_{a}$ is the rank of $P_{a}^{\left(n_{a}\right)}$. The projectors satisfy $P_{a}^{\left(n_{a}\right)} P_{b}^{\left(n_{b}\right)}=\delta_{a b} P_{i}^{\left(n_{a}\right)}$ and $\sum_{a=1}^{n} P_{a}^{\left(n_{a}\right)}=\mathbb{I}_{N+1}$. A state $\omega_{a}$ which satisfies

$$
\omega_{a}\left(P_{a}^{\left(n_{a}\right)} \hat{s}_{i} \hat{s}_{i} P_{a}^{\left(n_{a}\right)}\right)=s^{(a)}\left(s^{(a)}+1\right)
$$

gives us the $\mathbb{H}_{G N S}$ corresponding to the reducible representation $s^{(1)} \oplus s^{(2)} \cdots \oplus s^{(n)}$. The algebra $\mathcal{A}$ splits into $\sum_{a} \mathcal{A}^{(a)}$.

If all the $s^{(a)}$ are distinct, then the projectors $P_{a}^{\left(n_{a}\right)}$ are unique (upto unitary equivalence). This is not so when some of the $s^{(a)}(>0)$ are repeated. Let us illustrate this with an example. Consider the situation when $s^{(1)}=s^{(2)}=s>0$. Let us label the states by $\left.\mid s, s_{3} ; \alpha\right),\left|s_{3}\right| \leq s, \alpha=1,2$. The projector $P_{\alpha}$ is given by

$$
\left.P_{\alpha}=\sum_{s_{3}=-s}^{s} \mid s, s_{3} ; \alpha\right)\left(s, s_{3} ; \alpha \mid\right.
$$

These projectors are not unique. Under the transformation

$$
\left.\left.\mid s, s_{3} ; \alpha\right) \rightarrow \sum_{\alpha} u_{\beta \alpha} \mid s, s_{3} ; \alpha\right), \quad u \in \mathrm{U}(2)
$$

it is easy to check that

$$
\left.P_{\alpha} \rightarrow P[u ; \alpha]=\sum_{s_{3}=-s}^{s} \sum_{\beta, \gamma} u_{\gamma \alpha}^{\dagger} u_{\alpha \beta} \mid s, s_{3} ; \beta\right)\left(s, s_{3} ; \gamma \mid\right.
$$

is still a projector but it does not project to the fixed subspace $\left.\left\{\mid s, s_{3} ; \alpha\right)\right\}$.

It is important to recognise that these projectors are not elements of the observable algebra: there is no canonical construction of $P_{\alpha}$ using only the elements of the algebra $\mathcal{A}$.

The algebra $(3.22)$ is a $(4 n+4)$-dimensional reducible representation of $\mathrm{SU}(2)$ : it contains four identical copies of the $(n+1)$-dimensional irreducible representation. The carrier space of the reducible representation can be splited as (3.24). The states in $\mathcal{G}_{n}$ are labelled as

$$
\left.\mid s, s_{3} ; \alpha\right),
$$

where $\alpha=e e, e o, o e, o o$ labels the subspaces $F_{2 n}^{e e}, F_{2 n+1}^{e o}, F_{2 n+1}^{o e}$ and $F_{2 n+2}^{o o}$ respectively. For example in $\mathcal{G}_{1}, s=\frac{1}{2}$ and the states

$$
\left.\left.|2,0\rangle=\mid s=\frac{1}{2}, s_{3}=\frac{1}{2} ; e e\right), \quad|3,0\rangle=\mid s=\frac{1}{2}, s_{3}=\frac{1}{2} ; o e\right)
$$

belong to the irreducible subspaces $\mathcal{F}_{2}^{e e}$ and $\mathcal{F}_{3}^{o e}$ respectively.

The projectors $\Lambda^{\alpha}$ projects to these irreps. To indentify the specific irreps that a state belongs to, we need to know

$$
\left(s, s_{3} ; \alpha\left|\Lambda^{\beta}\right| s, s_{3} ; \alpha\right)=\delta_{\alpha \beta}
$$


But these projectors are not unique. $\Lambda^{\alpha}$ are given in terms of the $\hat{N}_{1}\left(=\hat{a}_{1}^{\dagger} \hat{a}_{1}\right)$ and $\hat{N}_{2}$ $\left(=\hat{a}_{2}^{\dagger} \hat{a}_{2}\right)$. But $\hat{N}_{1}$ and $\hat{N}_{2}$, and hence the projectors $\Lambda^{\alpha}$, are not elements of the algebra of observables (3.22).

As all the irreps are identical, the expectation value of any element $A$ of the algebra of observables in all the irreps are the same:

$$
\omega_{\alpha}(A)=\sum_{s_{3}} \sum_{s_{3}^{\prime}}\left(s, s_{3} ; \alpha|A| s, s_{3}^{\prime} ; \alpha\right), \quad \forall \alpha .
$$

Therefore with a probability vector $\lambda_{\alpha}\left(0 \leq \lambda_{\alpha} \leq 1, \quad \sum_{\alpha} \lambda_{\alpha}=1\right)$, we can define

$$
\omega(A)=\sum_{\alpha} \lambda_{\alpha} \omega_{\alpha}(A)=\omega_{\alpha}(A), \quad \forall \alpha .
$$

For any transformation

$$
\left.\left.\mid s, s_{3}: \beta\right)=\sum_{\alpha} u_{\beta \alpha} \mid s, s_{3}: \alpha\right)
$$

$u$ belongs to $\mathrm{U}(4)$ because the states are orthonormal and the spaces of the UIRs are invariant subspaces.

Further, under the transformation (5.10),

$$
\omega(A) \rightarrow \omega(A) .
$$

The state $\omega(A)$ remains invariant, and the system has $\mathrm{U}(4)$ gauge symmetry.

Under the transformation (5.10),

$$
\lambda_{\beta}(u)=\sum_{\alpha} \lambda_{\alpha}\left|u_{\alpha \beta}\right|^{2}
$$

The state $\omega_{\alpha}(A)$ can be written as

$$
\left.\omega_{\alpha}(A)=\operatorname{Tr}\left[\rho^{\alpha} A\right], \quad \rho^{\alpha} \equiv \sum_{s_{3}} p_{s_{3}} \mid s, s_{3}: \alpha\right)\left(s, s_{3}: \alpha \mid .\right.
$$

Similarly, $\omega(A)$ can be written as

$$
\omega(A)=\operatorname{Tr}[\rho A]
$$

The two density matrices are related as

$$
\rho \equiv \sum_{\alpha} \lambda_{\alpha} \rho^{\alpha}, \quad 0<\lambda_{\alpha}<1, \quad \sum_{\alpha} \lambda_{\alpha}=1 .
$$

The decomposition (5.15) is not unique. This non-uniqueness is parametrized by the unitary matrices $u \in \mathrm{U}(4)$. Therefore the state of the fuzzy sphere (3.22) is mixed which is invariant under the gauge group $\mathrm{U}(4)$.

The fuzzy sphere (3.22) being in a mixed state have dramatic consequences. In [16], it was shown that the fuzzy sphere in a reducible representation has higher energy than that 
in the irreducible representation, and that the reducible representations are metastable vacua. Our results show that this discussion needs to be refined: when the fuzzy sphere is in a reducible representation (with several identical copies of some particular irrep), then the corresponding quantum state is necessarily mixed. On the other hand the fuzzy sphere in the irreducible representation is in a pure state. The mixed states can never evolve to a pure state under a unitary time evolution, and a decay to the minimum energy configuration is not possible. Such a decay is possible only if one enlarges the algebra of observables.

Though $\operatorname{Tr}\left[\rho_{\alpha} A\right]$ is same in all the irreps, the von Neumann entropy is not:

$$
-\operatorname{Tr}\left[\rho_{\alpha} \log \rho_{\alpha}\right] \neq-\operatorname{Tr}\left[\rho_{\beta} \log \rho_{\beta}\right], \quad \alpha \neq \beta .
$$

This ambiguity in the definition leads to an intrinsic non-zero entropy in the mixed state. For the density matrix defined in (5.15), the von Neumann entropy is given by

$$
S=-\sum_{\alpha} \lambda_{\alpha}(u) \log \lambda_{\alpha}(u)
$$

The map $\lambda_{\alpha} \rightarrow \lambda_{\alpha}(u)$ is a Markovian:

$$
\lambda_{\beta}(u)=\sum_{\alpha} \lambda_{\alpha} T_{\alpha \beta}
$$

and the matrix $T$ satisfying

$$
T_{\alpha \beta}=\left|u_{\alpha \beta}\right|^{2} \geq 0, \quad \sum_{\alpha} T_{\alpha \beta}=1, \quad \sum_{\beta} T_{\alpha \beta}=1,
$$

is a doubly stochastic matrix. Being stochastic, it guarantees that the Markov process is irreversible and that the entropy of the system is driven to its maximum value. The entropy is maximized when $\lambda_{e e}=\lambda_{e o}=\lambda_{o e}=\lambda_{o o}=\frac{1}{4}$ and

$$
S_{\max }=2 \log 2 .
$$

The above formula for $S_{\max }$ is for the specific situation when the $S_{F}^{2}$ algebra has four identical irreps. The more general formula is derived later.

\subsection{Monopole states are mixed}

We showed in section 4 that fuzzy monopoles are described by the $\mathrm{SU}(2)$-representation

$$
[k \oplus(k+1) \cdots \oplus J] \oplus[k \oplus(k+1) \cdots \oplus J] \cdots \quad 16 \text { copies } .
$$

The states in distinct irreps above belong to different super-selection sectors. But the states belonging to the identical representations (say, $j=k$ in copy- 1 and $j=k$ in copy-2) cannot be distinguished by any observable of the algebra, because the projectors are not elements of this algebra. These states are 16-fold degenerate with a U(16) gauge symmetry, and are mixed. As before, we can write a density matrix

$$
\tilde{\rho}=\sum_{\mu} \tilde{\lambda}_{\mu}(\tilde{u}) \tilde{\rho}^{\mu}, \quad u \in \mathrm{U}(16), \quad \mu=e e \rightarrow e e, e e \rightarrow e o \cdots
$$


The entropy of this mixed state is

$$
\tilde{S}=\sum_{\mu}-\tilde{\lambda}_{\mu} \log \tilde{\lambda}_{\mu}
$$

The doubly stochastic process drives the system to a configuration with maximum entropy

$$
\tilde{S}_{\max }=4 \log 2 .
$$

More generally, we can consider the oscillators (3.7)

$$
\hat{b}_{1}^{\left(K_{1}\right)}=\sum_{i=0}^{K_{1}-1} \hat{b}_{1}^{i} \Lambda_{1}^{i}, \quad \hat{b}_{2}^{\left(K_{2}\right)}=\sum_{i=0}^{K_{2}-1} \hat{b}_{2}^{i} \Lambda_{2}^{i}
$$

where $K_{1}$ and $K_{2}$ are natural numbers.

When $K_{1} K_{2}>1$, the representations of the SU(2) algebra (3.22) are in general reducible and contain identical $K_{1} K_{2}$ irreps. Each state is $K_{1} K_{2}$-fold degenerate. The projectors $\Lambda_{1}^{i}$ and $\Lambda_{2}^{i}$ are not elements of the observable algebra and hence cannot be used distinguish between the irreps. As a result, the fuzzy sphere is in a mixed state and the maximum value of its entropy is

$$
S_{\max }=\log \left(K_{1} K_{2}\right) .
$$

A similar analysis holds for the fuzzy monopoles as well. The monopole bundle corresponds to a quantum mixed state, with von Neumann entropy

$$
\tilde{S}=\sum_{\mu=1}^{\left(K_{1} K_{2}\right)^{2}}-\tilde{\lambda}_{\mu} \log \tilde{\lambda}_{\mu}
$$

The entropy is maximized when $\tilde{\lambda}_{\mu}=\frac{1}{\left(K_{1} K_{2}\right)^{2}}$ :

$$
\tilde{S}_{\max }=\log \left(K_{1} K_{2}\right)^{2}=2 \log \left(K_{1} K_{2}\right) .
$$

\section{A Basis vectors for $\mathcal{H}_{n l}$}

Let $\mathcal{H}_{n l}^{e e \rightarrow e e}$ be the space of maps $\Phi^{e e \rightarrow e e}: \mathcal{F}_{2 n}^{e e} \rightarrow \mathcal{F}_{2 l}^{e e} . \mathcal{H}_{n l}^{e e \rightarrow e e}$ is also a bimodule which is a subset of $\mathcal{H}_{n l}$. In this subspace, the generators of $\mathrm{SU}(2)$ act as

$$
\hat{S}_{i} \Phi \equiv \hat{s}_{i}^{\left(l^{e e}\right)} \Phi^{e e \rightarrow e e}-\Phi^{e e \rightarrow e e} \hat{s}_{i}^{\left(n^{e e}\right)}, \quad \Phi^{e e \rightarrow e e} \in \mathcal{H}_{n l}^{e e \rightarrow e e} .
$$

where $\hat{s}_{i}^{\left(n^{e e}\right)}$ is the restriction of $\hat{s}_{i}$ in $\mathcal{F}_{2 n}^{e e}$ :

$$
\hat{s}_{+}^{e e}=\hat{b}_{1}^{e \dagger} \hat{b}_{2}^{e}, \quad \hat{s}_{-}^{e e}=\hat{b}_{2}^{e \dagger} \hat{b}_{1}^{e}, \quad \hat{s}_{3}^{e e}=\frac{1}{2}\left(\hat{b}_{1}^{e \dagger} \hat{b}_{1}^{e}-\hat{b}_{1}^{e \dagger} \hat{b}_{2}^{e}\right) .
$$

Let us consider the operator $\hat{f}_{1}=\left(\hat{b}_{1+}^{\dagger}\right)^{\tilde{l}}\left(\hat{b}_{2+}\right)^{\tilde{n}} \Lambda_{e e}$ :

$$
\begin{aligned}
& \hat{f}_{1}: \mathcal{F}_{2 n}^{e e} \rightarrow \mathcal{F}_{2 l}^{e e} \quad \text { if } \quad \tilde{l}-\tilde{n}=l-n, \quad 0 \leq \tilde{n} \leq n \\
& \text { with } k=\frac{l-n}{2} \quad \text { and } \quad J=\frac{l+n}{2} .
\end{aligned}
$$


It is easy to check that

$$
\begin{aligned}
\hat{S}_{+} \hat{f} \equiv\left[\hat{s}_{+}^{e e}, \hat{f}_{1}\right]=0 \\
\hat{S}_{3} \hat{f} \equiv\left[\hat{s}_{3}^{e e}, \hat{f}_{1}\right]=\frac{1}{2}(\tilde{l}+\tilde{n}) \hat{f}_{1} .
\end{aligned}
$$

So $\hat{f}_{1}$ is the highest weight function belonging to the spin- $j$ representation:

$$
j=\frac{1}{2}(\tilde{l}+\tilde{n}) .
$$

Let us denote this highest weight function by $\left(\Psi^{e e \rightarrow e e}\right)_{j j}^{J k}$ :

$$
\left(\Psi^{e e \rightarrow e e}\right)_{j j}^{J k}=\left(\hat{b}_{1}^{e \dagger}\right)^{\tilde{l}}\left(\hat{b}_{2}^{e}\right)^{\tilde{n}} \Lambda_{e e}
$$

The lower weight functions $\left(\Psi^{e e \rightarrow e e}\right)_{j m}^{J k}$ can be obatined by repeated action of the lowering operator:

$$
\left(\hat{S}_{-}\right)^{j-m}\left(\Psi^{e e \rightarrow e e}\right)_{j j}^{J k}=c_{j m}^{J k}\left(\Psi^{e e \rightarrow e e}\right)_{j m}^{J k}, \quad c_{j m}^{J k} \in \mathbb{C}
$$

where $\hat{S}_{-} \equiv\left[\hat{s}_{+}^{e e},\right]$.

The range of $j$ can easily be computed but putting the suitable values of $\tilde{n}$ and $\tilde{l}$ :

$$
j=k, k+1, k+2 \ldots J
$$

Open Access. This article is distributed under the terms of the Creative Commons Attribution License (CC-BY 4.0), which permits any use, distribution and reproduction in any medium, provided the original author(s) and source are credited.

\section{References}

[1] J. Hoppe, Quantum theory of a massless relativistic surface and a two-dimensional bound state problem, Ph.D. thesis, M.I.T., Cambridge U.S.A. (1982).

[2] J. Madore, The fuzzy sphere, Class. Quant. Grav. 9 (1992) 69 [InSPIRE].

[3] H. Grosse, C. Klimčík and P. Prešnajder, Topologically nontrivial field configurations in noncommutative geometry, Commun. Math. Phys. 178 (1996) 507 [hep-th/9510083] [INSPIRE].

[4] H. Grosse, C. Klimčík and P. Prešnajder, Field theory on a supersymmetric lattice, Commun. Math. Phys. 185 (1997) 155 [hep-th/9507074] [INSPIRE].

[5] H. Grosse, C. Klimčík and P. Prešnajder, On finite $4 D$ quantum field theory in noncommutative geometry, Commun. Math. Phys. 180 (1996) 429 [hep-th/9602115] [INSPIRE].

[6] M.R. Douglas and C.M. Hull, D-branes and the noncommutative torus, JHEP 02 (1998) 008 [hep-th/9711165] [INSPIRE].

[7] S. Baez, A.P. Balachandran, B. Ydri and S. Vaidya, Monopoles and solitons in fuzzy physics, Commun. Math. Phys. 208 (2000) 787 [hep-th/9811169] [INSPIRE]. 
[8] A.P. Balachandran and S. Vaidya, Instantons and chiral anomaly in fuzzy physics, Int. J. Mod. Phys. A 16 (2001) 17 [hep-th/9910129] [INSPIRE].

[9] S. Vaidya, Scalar multi solitons on the fuzzy sphere, JHEP 01 (2002) 011 [hep-th/0109102] [INSPIRE].

[10] N. Acharyya and S. Vaidya, Monopoles on $S_{F}^{2}$ from the fuzzy conifold, JHEP 06 (2013) 034 [arXiv: 1302.2754] [INSPIRE].

[11] A. Basu and J.A. Harvey, The M2-M5 brane system and a generalized Nahm's equation, Nucl. Phys. B 713 (2005) 136 [hep-th/0412310] [INSPIRE].

[12] H. Nastase, C. Papageorgakis and S. Ramgoolam, The fuzzy $S^{2}$ structure of M2-M5 systems in ABJM membrane theories, JHEP 05 (2009) 123 [arXiv:0903.3966] [INSPIRE].

[13] A.Y. Alekseev, A. Recknagel and V. Schomerus, Brane dynamics in background fluxes and noncommutative geometry, JHEP 05 (2000) 010 [hep-th/0003187] [INSPIRE].

[14] K. Hashimoto and K. Krasnov, D-brane solutions in noncommutative gauge theory on fuzzy sphere, Phys. Rev. D 64 (2001) 046007 [hep-th/0101145] [INSPIRE].

[15] D. Tomino, $N=23 D$ matrix integral with Myers term, JHEP 01 (2004) 062 [hep-th/0309264] [INSPIRE].

[16] R.C. Myers, Dielectric branes, JHEP 12 (1999) 022 [hep-th/9910053] [INSPIRE].

[17] J. Polchinski and M.J. Strassler, The string dual of a confining four-dimensional gauge theory, hep-th/0003136 [INSPIRE].

[18] D.P. Jatkar, G. Mandal, S.R. Wadia and K.P. Yogendran, Matrix dynamics of fuzzy spheres, JHEP 01 (2002) 039 [hep-th/0110172] [INSPIRE].

[19] Y. Kimura, Myers effect and tachyon condensation, Nucl. Phys. B 692 (2004) 394 [hep-th/0309082] [INSPIRE].

[20] R.A. Brandt and O.W. Greenberg, Generalized Bose operators in the Fock space of a single Bose operator, J. Math. Phys. 10 (1969) 1168.

[21] N. Acharyya, N. Chandra and S. Vaidya, Noncommutative vortices and instantons from generalized Bose operators, JHEP 12 (2011) 110 [arXiv:1109.3703] [INSPIRE].

[22] A.P. Balachandran, A.R. de Queiroz and S. Vaidya, Entropy of quantum states: ambiguities, Eur. Phys. J. Plus 128 (2013) 112 [arXiv:1212.1239] [INSPIRE].

[23] A.P. Balachandran, A.R. de Queiroz and S. Vaidya, Quantum entropic ambiguities: ethylene, Phys. Rev. D 88 (2013) 025001 [arXiv: 1302.4924] [inSPIRE].

[24] A.P. Balachandran, S. Kurkcuoglu and S. Vaidya, Lectures on fuzzy and fuzzy SUSY physics, World Scientific, Singapore (2007) [hep-th/0511114] [INSPIRE].

[25] H. Grosse and J. Madore, A noncommutative version of the Schwinger model, Phys. Lett. B 283 (1992) 218 [INSPIRE].

[26] J. Katriel and G. Adam, Equivalence of different representations of the generalized Bose operator, J. Math. Phys. 12 (1971) 1352. 